\title{
TITLE:
}

\section{ELLIPTIC BOUNDARY VALUE PROBLEMS IN THE SPACE OF DISTRIBUTIONS(Microlocal Geometry)}

$\operatorname{AUTHOR}(\mathrm{S}):$

ANDRONIKOF, E.; TOSE, N.

\section{CITATION:}

ANDRONIKOF, E.... [et al]. ELLIPTIC BOUNDARY VALUE PROBLEMS IN THE SPACE OF DISTRIBUTIONS(Microlocal Geometry). 数理解析研究所講究録 1993, 845: 13-18

ISSUE DATE:

1993-06

URL:

http://hdl.handle.net/2433/83623

RIGHT: 


\title{
ELLIPTIC BOUNDARY VALUE PROBLEMS \\ IN THE SPACE OF DISTRIBUTIONS
}

\author{
E. ANDRONIKof (UNIV. PARIS XIII) \\ AND

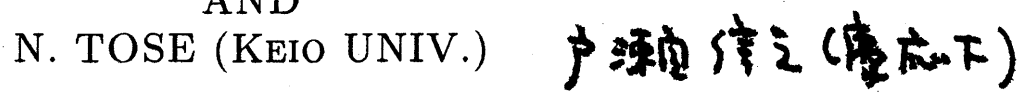

\section{Introduction}

Elliptic boundary value problems have their own long history. For the general system they were, however, first clearly fomulated microlocally by M. Kashiwara and T. Kawai [K-K]. Their theorem has enjoyed many applications, for example, to solvability of operators of simple characteristics, hypoelliptic operators, and tangential Cauchy-Riemann systems. The theorem does not give, however, much information if we restrict ourselves in the space of distributions. This note aims at giving an analogous theorem of KashiwaraKawai type in case function spaces are tempered. See Theorem 3 in Section 1 for the main theorem. By this theorem, we can obtain many application to distribution boundary values of holomorphic functions (e.g. M. Uchida[U]). The result of this note was obtained while the second author was staying in Univ. de Paris VI and Univ. Paris XIII. 
E. ANDRONIKOF (UNIV. PARIS XIII) AND N. TOSE (KEIO UNIV.)

\section{Main theorem}

Let $M$ be a real analytic manifolfd of dimension $n$ with a complex neighborhood $X$. Let $\mathcal{M}$ be a coherent $\mathcal{D}_{X}$ module on $X$ and assume that $\mathcal{M}$ is elliptic on $M$, i.e.

$$
\operatorname{char}(\mathcal{M}) \cap T_{M}^{*} X \subset T_{X}^{*} X
$$

Let $N$ be a real analytic submanifold of $M$ of codimension $d \geq 1$ in $M$, and $Y$ be a complexification of $N$ in $X$. We assume that $Y$ is non-characteristic for $\mathcal{M}$, i.e.

$$
\operatorname{char}(\mathcal{M}) \cap T_{Y}^{*} X \subset T_{X}^{*} X
$$

In this situation, we have the canonical morphisms

$$
T_{N}^{*} M \underset{\rho}{\longleftarrow} T_{N}^{*} X \underset{\varpi}{\simeq} T_{N}^{*} X .
$$

Under the above notation we have

THEOREM 1. The natural morphism

$$
\mathbf{R} \rho_{*} \underline{\operatorname{Rom}}_{\mathcal{D}_{X}}\left(\mathcal{M}, \mathcal{C}_{N \mid X}^{f}\right) \leftarrow \underline{\operatorname{Rom}}_{\mathcal{D}_{X}}\left(\mathcal{M}, T-\mu_{N}\left(\mathcal{D} b_{M}\right)\right) \otimes o r_{N / M}
$$

is an isomorphism.

In the above therem or $N / M$ denotes the relativs orientation sheaf of $N$ in $M$. The sheaf $\mathcal{C}_{N \mid X}^{f}$ on $T_{N}^{*} X$ is the tempered version of $\mathcal{C}_{N \mid X}$ and is given, with the tempered microlocalization due to E. Andronikof[An], by

$$
\mathcal{C}_{N \mid X}^{f}:=T-\mu_{N}\left(\mathcal{O}_{X}\right) \otimes \operatorname{or}_{M}[n]
$$

We remark that the above object in the derived category is concentrated in degree 0 . For a point $\stackrel{\circ}{x} \in T_{N}^{*} X$, the stalk of $\mathcal{C}_{N \mid X}^{f}$ at $\stackrel{\circ}{x}$ is given, with the aid of local cohomology with bounds, by

$$
\mathcal{C}_{N \mid X, \stackrel{\circ}{x}}^{f} \simeq \underline{\lim }_{[Z]}^{n}\left(\mathcal{O}_{X}\right)_{\pi_{X}(\stackrel{\circ}{x})} .
$$

Here $\pi_{X}$ denotes the projection $\pi_{X}: T^{*} X \longrightarrow X$ and the inductive limit is taken for all closed subanalytic sets $Z$ in $X$ satisfying the property

$$
C_{N}(Z)_{\pi_{X}(\stackrel{\circ}{x})} \subset\left\{v \in T_{N} X ;<\stackrel{\circ}{x}, v><0\right\} \cup\{0\}
$$


ELLIPTIC BOUNDARY VALUE PROBLEMS IN THE SPACE OF DISTRIBUTIONS

Refer here to Kashiwara-Schapira[K-S2] for the notion of normal cones $C_{N}(\cdot)$. The sheaf $T-\mu_{N}\left(\mathcal{D} b_{M}\right)$ on $T_{N}^{*} M$ is also constructed by E. Andronikof[An]. We just explain that its stalk at $\stackrel{\circ}{x} \in T_{N}^{*} M$ is given by the isomorphism

$$
T-\mu_{N}\left(\mathcal{D} b_{M}\right)_{\stackrel{\circ}{x}} \simeq \lim _{Z} \Gamma_{Z}\left(\mathcal{D} b_{M}\right)_{\pi_{M}(\stackrel{\circ}{)})} .
$$

Here the inductive limit is taken for any closed subanalytic set $Z$ in $M$ with the property

$$
C_{N}(Z)_{\pi_{M}(\stackrel{\circ}{x})} \subset\left\{v \in T_{N} M ;<\stackrel{\circ}{x}, v><0\right\} \cup\{0\}
$$

$\left(\pi_{M}: T^{*} M \longrightarrow M\right)$

Next we give another theorem, which is analogous to Theorem 6.3.1 of KashiwaraShapira [K-S1] (refer also to Kashiwara-Kawai[K-K] where we find the theorem of [K-S1] in its original form).

THEOREM 2. Let $\tilde{\mathcal{M}}=\mathcal{E}_{X} \otimes_{\pi_{X}^{-1} \mathcal{D}_{X}} \pi_{X}^{-1} \mathcal{M}$. Then the natural morphism

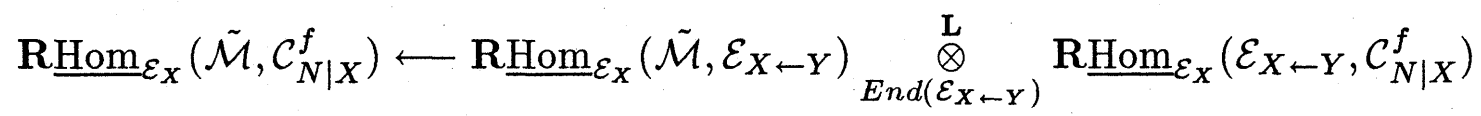

is an isomorphism outside of $T_{N}^{*} X \cap T_{Y}^{*} X$. This entails an isomorphism

$$
\underline{\mathbf{R o m}}_{\mathcal{E}_{X}}\left(\mathcal{M}, \mathcal{C}_{N \mid X}^{f}\right) \simeq \underline{\mathbf{R o m}}_{\mathcal{E}_{X}}\left(\mathcal{M}, \mathcal{E}_{X \leftarrow Y}\right) \otimes_{p^{-1} \mathcal{E}_{Y}}^{\mathbf{L}} p^{-1} \mathcal{C}_{N}^{f}
$$

on $T_{N}^{*} X \backslash T_{Y}^{*} X$ where $p$ is the canonical morphism

$$
p: T_{N}^{*} X \backslash T_{Y}^{*} X \longrightarrow T_{N}^{*} Y .
$$

In the above theorem, the object $\mathcal{C}_{N}^{f}$ on $T_{N}^{*} Y$ is the sheaf of temperate microfunctions. This is a subsheaf of $\mathcal{C}_{N}$ and describes microlocal analytic singularities of distributions on $N$. By the notation of E. Andronikof[An], this sheaf is defined as

$$
\mathcal{C}_{N}^{f}:=T-\mu_{N}\left(\mathcal{O}_{Y}\right)[n-d] \otimes o r_{N / Y}
$$

The proof of this theorem is essentially the same as in Theorem 6.3.1 of [K-S1] and relies on the division theorem of temperate microfunctions with holomorphic parameters with respect to microdifferential operators. We also remark that only the non-charactericity of $Y$ is utilized in its proof.

By combining the above theorems into one, we get the main theorem of this note. Let $q$ denote the restriction of $\rho$ to $\stackrel{\circ}{T}_{N}^{*} X \backslash T_{M}^{*} X ; q: \stackrel{\circ}{T}_{N}^{*} X \backslash T_{M}^{*} X \longrightarrow T_{N}^{*} M$ and $p$ the projection $\stackrel{\circ}{T}_{N}^{*} X \backslash T_{Y}^{*} X \longrightarrow \stackrel{\circ}{T}_{N}^{*} Y$. Then we have 
E. ANDRONIKOF (UNIV. PARIS XIII) AND N. TOSE (KEIO UNIV.)

THEOREM 3. We have a canonical isomorphism on $\stackrel{\circ}{T}_{N}^{*} Y$

$\mathbf{R} q_{*}\left(\mathbf{R H o m}_{\mathcal{E}_{X}}\left(\tilde{\mathcal{M}},\left.\mathcal{E}_{X \leftarrow Y}\right|_{T_{N}^{*} X}\right) \underset{p^{-1} \mathcal{E}_{Y}}{\stackrel{\mathrm{L}}{H}} p^{-1} \mathcal{C}_{N}^{f}\right) \simeq \underline{\mathbf{R o m}}_{\mathcal{D}_{X}}\left(\mathcal{M}, T-\mu_{N}\left(\mathcal{D} b_{M}\right)\right) \otimes o r_{N / M}$

\section{Idea of Proof}

What is left to us is now to construct the morphism in Theorem 1 and to show it an isomorphism.

First we construct a commutative diagram

(A)

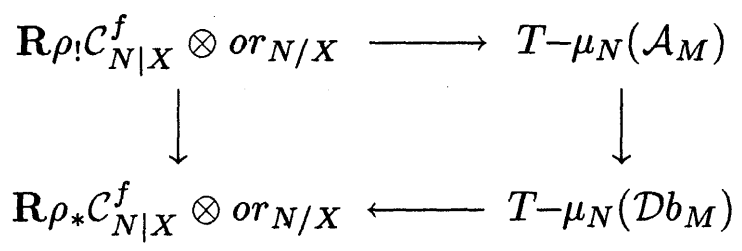

where $T-\mu_{N}\left(\mathcal{A}_{M}\right)$ is the tempered microlocalization of the sheaf $\mathcal{A}_{M}$ along $N$ and is constructed by E. Andronikof[A]. This object is the Fourier transform of the tempered specialization $T-\nu_{N}\left(\mathcal{A}_{M}\right)$ whose stalk at $\stackrel{\circ}{v} \in T_{N} M$ is given by

$$
T-\nu_{N}\left(\mathcal{A}_{M}\right)_{v} \simeq \lim _{U}\{u \in \mathcal{A}(U) ; u \text { is tempered on } M \text { as a distribution }\} .
$$

Here $U$ in the inductive limit ranges through any open subanalytic set in $M$ with the property

$$
\stackrel{\circ}{v} \notin C_{N}(M \backslash U)
$$

To construct (A), it is sufficient to construct its image by the inverse Fourier transformation

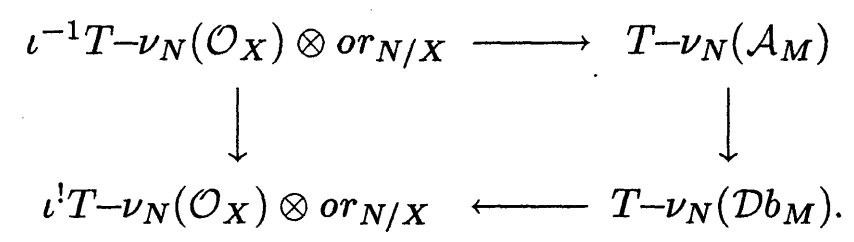

Here $\iota$ is the canonical embedding

$$
\iota: T_{N} M \longrightarrow T_{N} X
$$

and $T-\nu_{N}\left(\mathcal{O}_{X}\right)$ is the tempered specialization of the sheaf $\mathcal{O}_{X}$ along $N$, which is concentrated in degree 0 . The stalk of $T-\nu_{N}\left(\mathcal{O}_{X}\right)$ at $\stackrel{\circ}{v} \in T_{N} X$ is given by

$$
T-\nu_{N}\left(\mathcal{O}_{X}\right)_{\stackrel{\circ}{v}} \simeq \underline{\lim }_{U}\{u \in \mathcal{O}(U) ; u \text { can be extended to } X \text { as a distribution }\}
$$


ELLIPTIC BOUNDARY VALUE PROBLEMS IN THE SPACE OF DISTRIBUTIONS

where $U$ runs through all open subanalytic sets in $X$ with $\stackrel{0}{v} \notin C_{N}(M \backslash U)$. The diagram $\left(\mathrm{A}^{\prime}\right)$ can be constructed easily if we scrutinize the construction by E. Andronikof[An].

Next we apply $\underline{\mathbf{H o m}}_{\mathcal{D}_{\boldsymbol{X}}}(\mathcal{M}, \cdot)$ to the diagram $\left(\mathrm{A}^{\prime}\right)$ and obtain the commutative diagram

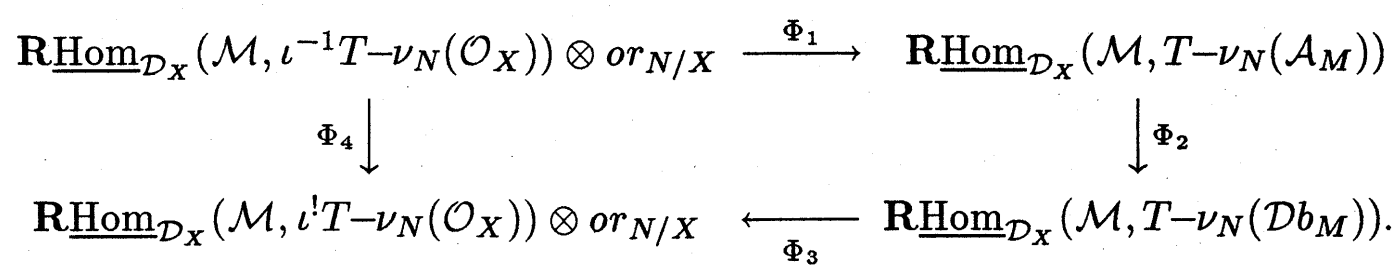

It is easy to see from the ellipticity of $\mathcal{M}$ that $\Phi_{4}$ and $\Phi_{2}$ are isomorphisms. (To show $\Phi_{4}$ is an isomorphism, it is easier to consider its image by Fourier transformation). Thus to prove that $\Phi_{3}$ and thus its image by Fourier transformation are isomorphisms, it suffices to show that $\Phi_{1}$ is an isomorphism. The problem for $\Phi_{1}$ can be reduced to the case where $\mathcal{M}$ is a single equation; i.e. $\mathcal{M}=\mathcal{D}_{X} / \mathcal{D}_{X} P$. Moreover it is sufficient to show that

$$
\underline{\operatorname{Hom}}_{\mathcal{D}_{X}}\left(\mathcal{D}_{X} / \mathcal{D}_{X} P, \iota^{-1} T-\nu_{M}\left(\mathcal{O}_{X}\right)\right) \otimes o r_{N / X} \longrightarrow \underline{\operatorname{Hom}}_{\mathcal{D}_{X}}\left(\mathcal{D}_{X} / \mathcal{D}_{X} P, T-\nu_{N}\left(\mathcal{A}_{M}\right)\right)
$$

is surjective. This problem can be solved by using the construction of the elementary solution of $P$ by means of Radon transformation and microdifferential operators. 
E. ANDRONIKOF (UNIV. PARIS XIII) AND N. TOSE (KEIO UNIV.)

\section{REFERENCES}

[An] Andronikof, E., Microlocalisation tempérée des distributions et des fonctions holomorphes I, II. C.R. Acad. Sci. t.303, 347-350 (1986) et t.304, $\mathrm{n}^{\circ} 17,511-514$ (1987). see also Thèse d'Etat, Paris-Nord, (juin 1987) and paper to appear.

[K-K] Kashiwara M. and T. Kawai, On the Boundary Value Problems for Elliptic Systems of Linear Differential Equations I, II, Proc. Japan Academy 48, pp. 712-715 (1972), 49, pp. 164-168 (1973).

[K-S1] Kashiwara, M. and P. Schapira, Microhyperbolic Systems, Acta Math. 142, pp. 1-55 (1974).

[K-S2] — MICROLOCAL STUDY of SHEAVES, Astérisque 128 (1985).

[U] Uchida, M., in these proceedings.

E. Andronikof

Département de Mathématiques, Univ. Paris XIII

93430 Villetaneuse, France

N. Tose

Mathematics, General Education, Keio Univ.

4-1-1 Hiyoshi, Yokohama 223, Japan 